\title{
Comunicação terapêutica em Enfermagem: instrumento essencial do cuidado
}

\author{
Therapeutic communication in Nursing: essential instrument of care
}

Comunicación terapéutica en Enfermería: herramienta esencial de la atención

\author{
Alexandra Carvalho Pontes', Ilse Maria Tigre Arruda Leitão', Islane Costa Ramos' \\ 'Universidade Estadual do Ceará, Departamento de Enfermagem. Fortaleza, CE
}

Submissão: 01/10/2007

Aprovação: 12/12/2007

\section{RESUMO}

O objetivo deste estudo foi analisar o processo de comunicação terapêutica desenvolvida por enfermeiros numa unidade de internação com base na teoria de Peplau. Pesquisa descritiva-exploratória realizada em um Hospital Público de Fortaleza, CE, em outubro e novembro de 2006. Participaram 14 pacientes e 12 enfermeiros, através de uma entrevista semi-estruturada. Os dados foram organizados em temáticas, discutidas com base na literatura. Os resultados revelam que, desde a admissão até a alta do paciente, há comunicação e interação, sendo desenvolvido um relacionamento interpessoal. Muitas vezes, contudo, essa comunicação não é como deveria ser, pois a enfermeira pouco prioriza em seu tempo de trabalho as visitas junto aos pacientes. Estes têm dificuldade de distinguir as enfermeiras dos outros membros da equipe, dificultando a fase de aproximação e o desenvolvimento de uma comunicação terapêutica.

Descritores: Comunicação; Enfermagem; Cuidado.

\section{ABSTRACT}

The objective of the study was to analyze the process of therapeutic communication as developed by nurses who work in hospital units according to Peplau's theory. It is a descriptive-exploratory research accomplished in the period of October and November of 2006 , of the Municipal district of Fortaleza, CE, Brazil in octuber and november, 2006. The participants of the study were 14 patients and 12 nurses, through a a semi-structured interview. Data were organized in thematic and discussed with literature review. Results demonstrated that since admission to hospital discharge, there is communication and interaction with a interpersonal relationship. Several times, however, that communication does not accur as it was supposed to, beacause nurse in her work does not priorize the visit to patients in her daily activities. Patients have difficulties to distinguish nurses from other halth care professionals, which difficults the approach and development of therapeutic communication.

Descriptors: Communication; Nursing; Care.

\section{RESUMEN}

El objetivo de esto estudio fue analizar el proceso de comunicación terapéutica desarrollada por enfermeros en una unidad de internación basada en la teoría de Peplau. Es una Investigación descriptiva-exploratoria realizada en un Hospital Público de FortalezaCE, de octubre y noviembre de 2006. Participaron 14 pacientes y 12 enfermeros, siendo realizada una entrevista semi-estructura. Los datos fueron organizados en temas, basada en la literatura. Los resultados señalaron que desde la admisión hasta la alta de paciente, hay comunicación e interacción, siendo desarrollada así una relación interpersonal. En la mayoría de las veces, por supuesto, esa comunicación no es como debería haber. Se constató que la enfermera no hace prioridad en su tiempo de trabajo a las visitas junto a los pacientes. Estos pocos diferencian a las enfermeras de los otros componentes del equipo de enfermería, lo Que dificulta la fase de aproximación entre ellos y el desarrollo de una comunicación terapéutica.

Descriptores: Comunicación; Enfermería; Atención. 


\section{INTRODUÇÃO}

A comunicação é um instrumento básico do cuidado em enfermagem. Ela está presente em todas as ações realizadas com o paciente, seja para orientar, informar, apoiar, confortar ou atender suas necessidades básicas. Como instrumento, a comunicação é uma das ferramentas Que o enfermeiro utiliza para desenvolver e aperfeiçoar o saber-fazer profissional ${ }^{(1)}$.

O papel do enfermeiro não se restringe a executar técnicas ou procedimentos e sim propor uma ação de cuidados abrangente, Que implica, entre outros aspectos, desenvolver a habilidade de comunicação. Deste modo, o uso da comunicação como instrumento básico do enfermeiro é um meio utilizado para atender as necessidades do paciente.

É pela comunicação Que as pessoas podem expressar o Que são, relacionar-se, satisfazer suas necessidades. Essa interação pode influenciar o comportamento das pessoas, Que reagirão com base em suas crenças, valores, história de vida e cultura ${ }^{(2)}$. Por isso, o relacionamento entre enfermeiro e paciente adQuire tanta importância no fenômeno de cuidar.

As mudanças no processo de trabalho de enfermagem influenciam a relação entre enfermeira e paciente, a Qual, ao longo dos tempos, vem se modificando gradativamente. Com a humanização da enfermagem, o paciente deixou de ser visto apenas como uma doença ou como um leito e passou a ser visto como um todo e de forma individualizada. Com isso, a enfermagem passou a identificar as necessidades básicas de cada paciente para poder agir sobre elas.

Para identificar essas necessidades, cada vez mais, o paciente se faz sujeito ativo nesse relacionamento. Mediante os vínculos estabelecidos, o trabalho da enfermagem é otimizado e o paciente é beneficiado com isso. É pela comunicação estabelecida com o paciente, Que se pode compreendê-lo holisticamente, isto é, seu modo de pensar, sentir e agir ${ }^{(3)}$.

Cada paciente e família possuem a chave para o cuidado de enfermagem efetivo, pois, Quando a confiança é estabelecida, informações são proporcionadas e a pessoa é encorajada a tomar parte ativa na maximização de sua capacidade de funcionamento, pois a enfermeira cria estratégias para atingir a saúde ideal e abre a porta à satisfação do paciente e à eficiência do cuidado à saúde ${ }^{(4)}$.

Esse relacionamento, no entanto, não deve ser uma atitude mecânica, como freqüentemente ocorre. O paciente não deve ser visto apenas como um objeto de trabalho para a equipe de enfermagem, pois, assim, somente algumas necessidades dele serão satisfeitas. O relacionamento terapêutico depende do comportamento e atitudes de cada profissional.

Conhecendo a importância da comunicação terapêutica e suas influências no restabelecimento da saúde dos pacientes, deve-se avaliar se esta ocorre, de modo Que se considere, relevante realizar uma reflexão sobre as interfaces desse cuidado ao cliente hospitalizado, de forma a contribuir para a melhoria da Qualidade da assistência de enfermagem, sob o prisma do processo de comunicação. Assim, os resultados da pesquisa devem ser utilizados para promover maior reflexão dos enfermeiros em relação a trabalhar aspectos Que favoreçam o relacionamento interpessoal e, conseQüentemente, a comunicação terapêutica.

O conceito de comunicação terapêutica adaptado da teoria de Ruesch, consiste na habilidade do profissional em usar seu conhecimento sobre comunicação para ajudar a pessoa com tensão temporária a conviver com outras pessoas e ajustar-se ao Que não pode ser mudado e a superar os bloqueios à auto-realização, para enfrentar seus problemas ${ }^{(3)}$.

Assim, vê-se a comunicação como um processo Que pode ser utilizado como instrumento de ajuda terapêutica. Para tanto, o enfermeiro deve ter conhecimentos fundamentais sobre as bases teóricas da comunicação e adQuirir habilidades de relacionamento interpessoal para agir positivamente na assistência ao paciente. Para Que esta possa fluir bem, a enfermeira deve saber escutar, falar Quando necessário, dar abertura para realização de perguntas, ser honesto, mostrar respeito, dispensar tempo suficiente para a conversa e mostrar interesse, entre outras habilidades.

Esse tipo de comunicação facilita a identificação dos problemas por parte da enfermeira e permite melhor assistência ao paciente. $\mathrm{O}$ planejamento, o estabelecimento de metas e a seleção das intervenções apropriadas são essenciais à elaboração de um plano de cuidados, bem como a prestação de um atendimento Qualificado de enfermagem $^{(5)}$.

A comunicação não é simplesmente uma troca de mensagens entre a enfermeira e o paciente, mas é uma ação Que deve ser planejada e individualizada, não sendo realizada somente por impulsos e de forma intuitiva. Há diversos guias e técnicas Que podem ser utilizados para tornar terapêutica essa comunicação.

A enfermeira, a partir da comunicação desenvolvida com o paciente, identifica suas necessidades, informa sobre procedimentos ou situações Que ele deseja saber, promove o relacionamento do paciente com outros pacientes, com a equipe multiprofissonal ou com familiares, promove educação em saúde, troca de experiências e mudança de comportamentos, entre outros. Essas são algumas das funções da comunicação em Que a enfermeira pode estar envolvida, o Que não Quer dizer Que o paciente não possa ser também sujeito ativo dessas ações.

Portanto, este estudo tem como objetivo analisar o processo de comunicação terapêutica desenvolvido por enfermeiros numa unidade de internação, com base em Peplau.

\section{MARCO TEÓRICO}

Uma das teorias considerada como referência para a prática da enfermagem e, sobretudo, para o processo de comunicação em enfermagem, é a Teoria das Relações Interpessoais, desenvolvida por Hildegard E. Peplau, em 1952. A teórica visualizou o fenômeno de enfermagem como um processo interpessoal cujo foco principal está centralizado na enfermeira e no paciente. Neste sistema, ela pretende identificar conceitos e princípios Que dêem suporte às relações interpessoais processadas na prática da enfermagem, de modo Que as situações de cuidado possam ser transformadas em experiências de aprendizagem e crescimento ${ }^{(6)}$.

Com a publicação do seu livro Interpersonal Relations in Nursing: a conceptual frame of reference for psychodinamic nursing, em 1952. Hildegard Peplau introduziu um novo paradigma para a enfermagem, centrado nas relações interpessoais Que se processam entre enfermeiras e pacientes ${ }^{(7)}$.

Os elementos fundamentais da prática da enfermagem são os pacientes, a enfermeira e os acontecimentos Que envolvem ambos durante uma situação de cuidado. Eis, portanto, a importância de 
Peplau, ao tentar documentar o Que a enfermagem, de certa forma, já realiza Quando interage com o paciente, embora o faça, na maioria das vezes, de forma intuitiva.

A saúde precisa de uma definição clara e, após tê-la em mente, é possível ver Que a enfermeira participa com outros profissionais na organização de condições Que facilitam o movimento progressivo da personalidade e outros processos humanos. O processo interpessoal é operacionalmente definido em Quatro fases distintas: orientação, identificação, exploração e solução. Estas fases podem ser correlacionadas com as etapas tradicionais do processo de enfermagem $^{(6)}$.

Uma de tais Questões referia-se à compreensão do Que seria a enfermagem e em Que consistiria a sua prática. Em resposta a esse Questionamento, Peplau vislumbrou a enfermagem como um processo interpessoal, por meio do Qual enfermeira e paciente podem obter crescimento e desenvolvimento pessoais com o objetivo de promover a saúde $\mathrm{e}^{(7)}$.

Com base nessas proposições, Peplau traz em sua teoria a noção de crescimento pessoal, Que é compartilhado pela enfermeira e pelo paciente no relacionamento interpessoal desenvolvido nos atos de cuidar. A autora usou a expressão enfermagem psicodinâmica para descrever o relacionamento dinâmico entre enfermeira e paciente. No seu entendimento, a enfermagem psicodinâmica envolve reconhecer, esclarecer e estabelecer uma compreensão acerca do Que acontece Quando a enfermeira se relaciona de forma útil com o paciente ${ }^{(8)}$.

As etapas da enfermagem psicodinâmica desenvolvem-se tendo como base dois pressupostos: a atitude adotada pela enfermeira interfere diretamente no Que o paciente vai aprender durante o cuidado, ao longo de sua experiência, como doente; o auxílio ao desenvolvimento da personalidade e ao amadurecimento é uma função da enfermagem, Que exige o uso de princípios e métodos facilitadores e orientadores da solução dos problemas ou dificuldades interpessoais cotidianas ${ }^{(8)}$.

É necessário ressaltar Que o processo vai muito além das técnicas de enfermagem, Que poderão ser usadas ou não para resolver o problema do paciente, mas Que, por si só, não o levam a amadurecer. O objetivo da assistência de enfermagem é ajudar os indivíduos e a comunidade a produzir mudanças Que influenciem de forma positiva na sua saúde. Vale mencionar Que as metas a serem atingidas deverão ser estabelecidas pelo enfermeiro e pelo paciente, pois, caso os objetivos de ambos sejam desarmônicos, os resultados também o serão.

Ao incluir nos objetivos as mudanças em âmbito comunitário, Peplau evidencia o fato de Que reconhece o papel da família, da sociedade, da cultura e do ambiente nas mudanças, mesmo Que seja o ambiente hospitalar o contexto predominante na teoria. Também indica que um dos papéis Que a enfermeira pode desenvolver é o de fazer com que os cuidados de saúde possam ser conduzidos do hospital para a comunidade ${ }^{(7)}$.

Com o desenvolvimento de uma relação interpessoal no processo de cuidado com base em Peplau, a enfermeira tem a oportunidade de tornar a comunicação terapêutica mais adeQuada e eficaz.

\section{METODOLOGIA}

Pesquisa descritiva-exploratóra. O estudo exploratório busca a familiarização com o fenômeno, a percepção e a descoberta de idéias a respeito do assunto visado. Descreve detalhadamente a situação e procura descobrir as relações entre os seus elementos, considerando os mais diversos aspectos envolvidos ${ }^{(9)}$.

A coleta de dados foi realizada em outubro e novembro de 2006, em um hospital público, situado na cidade Fortaleza, CE. Tem capacidade instalada de 303 leitos de internação e média mensal de 1293 internações.

Participaram do estudo doze enfermeiras e quatorze pacientes, escolhidos aleatoriamente e que concordaram participar da pesquisa, sendo preenchido o Termo de Consentimento Livre e Esclarecido.

Foram utilizados como técnica de coleta de dados a entrevista semi-estruturada, o diário de campo para registro das observações e um roteiro de coleta junto ao prontuário.

A análise dos dados teve por base a Análise Temática, constitui uma das técnicas, onde se classificam os diversos elementos da comunicação, a partir de leituras Que permitem identificar o sentido, colocando em ordem as idéias expressas pelos entrevistados ${ }^{(10)}$.

Os aspectos éticos foram resguardados durante toda a investigação, tal como é previsto na Resolução 196/96, do Conselho Nacional de Saúde do Brasil( ${ }^{(1)}$. O projeto de pesQuisa foi aprovado pelo Comitê de Ética em Pesquisa do Hospital onde o estudo foi realizado.

\section{RESULTADOS E DISCUSSÃO}

\section{Compreendendo a Relação Terapêutica do Enfermeiro}

\section{Sistematização da Assistência de Enfermagem}

O processo de enfermagem foi desenvolvido como um modo para sistematizar os cuidados em enfermagem, melhorando sua eficácia e sua Qualidade. Facilita a forma de planejar e implementar os cuidados ao paciente de modo mais independente e eficiente. Quando os enfermeiros praticam os modelos do processo de enfermagem, os pacientes recebem cuidados altamente Qualificados em um mínimo de tempo e um máximo de eficiência ${ }^{(12)}$.

Das enfermeiras Que participaram da pesquisa, pôde-se constatar Que elas não utilizam a sistematização da assistência de enfermagem por não estar implantada no hospital onde trabalham ou por compreenderem Que somente deve ser utilizada em pacientes graves.

A gente não tá trabalhando com a sistematização. A gente até tentou, já fez um projeto (E6).

Não é implantada não. Aqui é uma unidade Que os pacientes não reQuer de cuidados especiais. São pacientes Que deambulam, Que verbalizam, não têm pacientes graves (E2).

Só dos pacientes graves. A gente vê o Que ta precisando, Quais são os cuidados pra gente pegar e organizar bem direitinho (E7).

Nestes depoimentos, se pode notar Que, para as enfermeiras, a sistematização da assistência de enfermagem só é necessária aos pacientes graves. Sabe-se, porém, Que o processo de enfermagem é um instrumento Que favorece o cuidado e organiza as condições 
necessárias para que o cuidado seja realizado, mesmo aos pacientes mais estáveis.

Não é aquilo que a gente esperava, mas eu posso dizer que sim. Estamos tentando implantar e estamos fazendo o que dá diante do nosso tempo. Nós ainda estamos iniciando os cuidados (E4).

A gente não tem implementado dentro dos padrões acadêmicos. Mas, o Que a gente faz é algo assim, um roteiro prático e não um roteiro sistematizado. Com impressos e tudo mais (E5).

Os processos de enfermagem, vinculados ou não a teorias de enfermagem, fundamentam-se no método de resolução científica de problemas e, por isso, suas fases propõem a coleta de informações, a definição do problema, a opção por uma solução, testando-a, avaliando seus resultados e tentando outras soluções se a primeira não tiver sido satisfatória ${ }^{(13)}$.

Observou-se Que, apesar do processo de enfermagem não estar implementado nas unidades de internação como uma rotina do serviço, algumas enfermeiras estão praticando, buscando, com isso, melhorar a assistência ao paciente.

Verificou-se também Que existe a justificativa de não realizar a sistematização da assistência de enfermagem com todos os pacientes, em virtude do excesso de atribuições das enfermeiras.

Só com os mais complicados. PorQue não tem condições de fazer com todos. Nós somos muito cobradas no sentido de dar conta dos pacientes. A gente então priorizar os exames, as admissões, altas, assistência a pacientes grave (EI).

O tempo ou a falta de tempo é uma Queixa geral e forte obstáculo, comprometendo, assim, a importância do processo de enfermagem, sem contar com problemas como número reduzido e preparo inadequado de pessoal Que compõe a equipe ${ }^{(14)}$.

Se, porém, o trabalho da enfermeira for guiado pelo processo de enfermagem, haverá um planejamento, e conseeüentemente facilitará a execução e a avaliação do plano de cuidados, valorizando o tempo e identificando possíveis problemas Que estejam impedindo o desenvolvimento deste processo, sejam recursos humanos ou técnicos.

A sistematização do cuidado deve estar registrada, de forma a proporcionar uma comunicação efetiva entre os membros da equipe de saúde e a avaliação da eficácia do cuidado prestado ao cliente, contribuindo para um melhor nível assistencial ${ }^{(8)}$.

Entende-se a importância do processo de enfermagem, tanto para o paciente como para o enfermeiro, destacando-se a idéia de Que o desenvolvimento de suas fases deve ser documentado no prontuário do paciente, salientando-se que essa atividade permite a todos os membros da equipe de saúde estar cientes das ações adotadas e resultados obtidos com os cuidados prestados ao paciente.

Percebe-se, de maneira geral, Que a sistematização da assistência de enfermagem não está sendo implementada nas unidades. $\mathrm{E}$ nota-se também falta de interesse e iniciativa por parte das enfermeiras. Sistematizar o cuidado é algo que todo enfermeiro sabe, mas não pratica, seja por falta de tempo, seja por falta de profissionais em número suficiente, seja por desconhecimento ou por não estar implantado o processo na instituição onde trabalham ${ }^{(13)}$

Os enfermeiros são a fonte principal de contato pessoal, íntimo e contínuo, com os pacientes, não obstante seu envolvimento com a tecnologia e com a burocracia hospitalar. São eles os responsáveis pela implementação do cuidado a cada paciente, individualmente, o Que lhes confere oportunidade de orientá-lo e de prestar-lhe informações completas, precisas e verdadeiras sobre os procedimentos que os integrantes da equipe de enfermagem, ou os outros profissionais da saúde, desempenharão com ele e para ele $^{(15)}$.

Não desenvolver o processo de enfermagem implica dificultar a comunicação terapêutica, pois se identificou o fato de Que as enfermeiras não planejam suas atividades; assim, não fazem o histórico de enfermagem, momento este de suma importância para a troca de informações, orientações, de desenvolver uma relação empática e de identificar os problemas, ou seja, de trabalhar o relacionamento interpessoal e assim facilitar a comunicação terapêutica.

\section{Comunicação Terapêutica e Relacionamento Interpessoal}

A comunicação enfermeiro-paciente é denominada comunicação terapêutica, porque tem a finalidade de identificar e atender as necessidades de saúde do paciente e contribuir para melhorar a prática de enfermagem, ao criar oportunidades de aprendizagem e despertar nos pacientes sentimentos de confiança, permitindo Que eles se sintam satisfeitos e seguros ${ }^{(3)}$.

Quando Questionadas sobre o Que entendiam por comunicação terapêutica, as enfermeiras responderam Que era uma conversa entre enfermeira e paciente. Pelos depoimentos, entretanto, viuse Que muitas vezes elas relacionam esse processo somente com o estado clínico de paciente.

Comunicação Que envolve o paciente e a enfermagem de acordo com a terapia Que ele necessita, para ajudar na terapêutica da doença $(\mathrm{EI})$.

A comunicação entre os profissionais de saúde sobre o paciente, algo para a melhora do paciente (E3).

É Quando conversamos com o paciente, identificamos problemas e após a conversa, o deixamos em melhores condições (E8).

Percebe-se Que as enfermeiras identificam que esse processo beneficia o paciente, mas, ao mesmo tempo, este não participa ativamente dele.

Um dos objetivos da assistência de enfermagem é levar o paciente a participar dos esQuemas terapêuticos. Esta participação depende dos processos de comunicação, a partir dos Quais se estabelecem as relações de confiança necessárias para o paciente diminuir o medo, a ansiedade e permitir, à pessoa fragilizada pela doença, lutar por seu restabelecimento com dignidade. Nesse sentido, os enfermeiros intensivistas, além da experiência no uso de tecnologias, e do suporte psicossocial, devem estar atentos ao processo de comunicação para Que o paciente possa suportar os efeitos da hospitalização, manter a percepção de si próprio e da sua realidade $^{(16)}$. 
Para os enfermeiros, a comunicação com o paciente é considerada fundamental, não apenas para a identificação de sinais, sintomas e problemas físicos, mas também para o desenvolvimento de uma interação terapêutica. Entender a comunicação humana como estratégia para a melhoria da Qualidade da assistência torna-se fundamental para o acontecimento desse processo.

A comunicação precisa ser considerada no seu contexto de ocorrência, ou seja: onde, como e Quando ocorre. Caso contrário, seu sentido pode ser prejudicado, razão pela Qual, na assistência à saúde, ela precisa ser planejada para cada interação e adequada a cada paciente. Nesse contexto, ela é parte das atividades do enfermeiro, porQue é empregada em situações como na entrevista, no exame físico, no planejamento da assistência, nas anotações dos prontuários e nas orientações aos indivíduos, famílias e comunidades. Disso decorre a importância de o enfermeiro ter consciência da forma como acontece o processo de comunicação e dos elementos Que o compõem.

Um dos instrumentos Que facilita a comunicação terapêutica é o bom relacionamento interpessoal, pois, segundo Peplau, este favorecerá a troca de informações a partir das estratégias utilizadas e da criação de um universo propicio à identicação dos problemas. O processo interpessoal é operacionalmente definido em Quatro fases distintas: orientação, identificação, exploração e solução. Estas fases distintas podem ser correlacionadas às etapas tradicionais do processo de enfermagem, ou seja, levantamento de dados, diagnósticos de enfermagem, planejamento e implementação de intervenções e avaliação, respectivamente ${ }^{(6)}$.

Eu acho Que é interagir com o paciente, com o Que ele tem, com o que pode acontecer, o tratamento dele, o Que vai ser feito e como pode ajudar (EI2).

Eu acho que toda comunicação que você faz com o paciente é necessária, é uma terapia mesmo. Quando você conversa, dá atenção, ele se transforma em outra pessoa. Você tem uma abertura com eles, Que eles se sentem melhor (EI I).

A capacidade da enfermeira para incentivar comunicação é muito importante, especialmente na investigação de problemas com o paciente e no encorajamento para expressar os seus sentimentos. É só no entendimento Que é comunicação terapêutica Que a enfermeira pode promover uma assistência de Qualidade e individualizada.

Porque através dessa conversa que desenvolvo o relacionamento interpessoal, contribuindo no cuidado do paciente (E4).

Com certeza. Porque é através do diálogo que você vai saber as coisas, não vai passar em branco, se você não perguntar eles não dizem (E6).

O cuidado é um ato profissional do enfermeiro e se expressa no relacionamento interpessoal; é por meio dessa interação que o plano e a comunicação terapêutica são efetivados.

A comunicação terapêutica permite a interação entre enfermeira e paciente e proporciona a oportunidade de se conseguir um relacionamento humano Que atinja os objetivos da assistência. $\mathrm{O}$ uso da comunicação terapêutica pelo enfermeiro aumenta a aceitação e o entendimento do paciente Quanto à realização dos procedimentos, diminuindo a ansiedade ${ }^{(17)}$.

Dessa forma, a comunicação é de suma importância na prática de enfermagem, pois permite ao profissional estabelecer um relacionamento interpessoal com os pacientes, ajudando-os na sua recuperação, pois por meio da comunicação criam-se condições para Que o profissional de enfermagem possa efetivar mudanças, no intento de promover o bem-estar do paciente.

Assim, desenvolver as habilidades de comunicação é de fundamental importância para os profissionais da área de saúde, em especial os enfermeiros, Que devem conhecer o significado das mensagens enviadas pelo paciente para então elaborar um plano assistencial adequado para atender as necessidades dele.

A comunicação é um ato criativo; não existe apenas um agente emissor ou receptor, mas uma troca entre as pessoas Que formam um sistema de interação e reação, isto é, um processo recíproco Que provoca mudanças na forma de sentir, pensar e atuar dos envolvidos.

\section{Compreensão do Relacionamento Terapêutico sob o Ponto de Vista do Paciente}

\section{Identificação da EQuipe de Enfermagem}

A fase inicial de apresentação diminui a tensão e a ansiedade em decorrência da redução da distância entre enfermeira e paciente. Ambos sentem-se mais à vontade um com o outro, possibilitando continuidade e sucesso dos cuidados à saúde. Observa-se, contudo, nas falas a seguir Que o enfermeiro não é reconhecido com facilidade pelos pacientes:

Nem percebo muito não. Eles se identificam, mas não conheço bem (PI).

Sei pouco Quem são os enfermeiros, são os auxiliares Que estão mais pertos e orienta. Eu não consigo saber e eles vem pouco falar com a gente (P2).

Elas Quase não conversam, eu só vejo Quando tem algum problema (P8).

Os pacientes expressam dificuldade para identificar o enfermeiro, pois revelam Que ele não está próximo deles, conversando, orientando, realizando visitas para avaliá-los e que freqüentemente se mantém distante, ficando, assim, difícil o estabelecimento de um vínculo entre eles Que favoreça a comunicação terapêutica.

\section{Comunicação Terapêutica e Relacionamento Interpessoal}

A relação terapêutica pode proporcionar o desenvolvimento do autocuidado no paciente. Dessa relação, pode surgir um interesse participativo do paciente, tornando-se um agente operante no seu processo saúde-doença e não um ser passivo. Ele passa a ter independência e a adQuirir responsabilidades na sua recuperação.

Ao serem Questionados como era a comunicação do enfermeiro, a maioria dos pacientes respondeu Que, no geral, eles tratam bem. Alguns não souberam responder, pois a enfermeira ainda não havia 
mantido contato com eles.

Ficam brincando com a gente, distraindo. Mas tem algumas que são sérias. Mas é o jeito da pessoa mesmo (P7).

Eles chegam alegres, satisfeitos. Dão bom dia. Pergunta como a gente tá, se tá sentindo alguma coisa. Só isso (P9).

Pelo relato dos pacientes, percebe-se Que algumas enfermeiras, conscientemente ou não, estão fazendo uso de técnicas de comunicação terapêutica e relacionamento interpessoal.

Ao receber o paciente calorosamente no início do contato, o profissional faz com Que ele se sinta valorizado e se entregue ao atendimento $^{(13)}$.

Tratando-se do relacionamento enfermeiro-cliente, o processo de comunicação precisa ser eficiente para viabilizar uma assistência humanizada e personalizada, de acordo com suas necessidades. Portanto, a interação com o cliente se caracteriza não só por uma relação de poder em Que este é submetido aos cuidados do enfermeiro, mas, também, por atitudes de sensibilidade, aceitação e empatia entre $\operatorname{ambos}^{(8)}$.

Mas tem umas Que falam aborrecidas e até a gente nem agüenta. Falam umas coisas e a gente fica até alterado, nem se interessa (PII).

Tratam a gente de forma distante, não se preocupam muito (PI4).

Algumas enfermeiras, com esse tipo de atitudes não terapêuticas, não conseguem assistir o paciente adequadamente, uma vez que eles não se sentem a vontade para expor seus problemas. Há os Que, por inibição e humildade, não expressam sua insatisfação, mas isso prejudica na recuperação ${ }^{(13)}$. Alguns pacientes sentem envergonhados de reclamar do Serviço da Enfermagem e do modo como são tratados, prejudicando, assim, a comunicação e, conseQüentemente a sua recuperação.

Não vem muitas vezes, não. Vem poucas vezes (P6).

É mais difícil as enfermeiras virem aQui. Elas ficam mais no posto (P7).

A enfermeira vem aqui no Quarto, olha e sai. Confere só os pacientes (P8).

Nota-se Que, dentre as atividades da enfermeira, pouco tempo é dispensado para a visita dos pacientes, ou seja, para a identificação dos problemas e avaliação dos resultados, algumas vezes chegando nem a acontecer. O estado do paciente é dinâmico e se não houver maior proximidade do enfermeiro, a continuidade e Qualidade do seu trabalho ficarão prejudicadas. Os pacientes precisam de tempo para estabelecer laços de confiança com o enfermeiro, adQuiridos com o relacionamento interpessoal adequado e que facilitem o processo de comunicação terapêutica, pois a visita de enfermagem é uma etapa fundamental para identificar as necessidades daQueles sob os seus cuidados.
É na da visita de enfermagem Que o enfermeiro estabelece a comunicação com o paciente, possibilitando o esclarecimento de dúvidas Quanto à evolução e prognóstico, aos procedimentos a serem realizados, normas e rotinas da instituição ou unidade de internação e estrutura física hospitalar, desempenhando um importante papel na redução dos Quadros de tensão e ansiedade Que repercutem no Quadro clínico do cliente.

Neste sentido, percebe-se Que o enfermeiro, ao utilizar estratégias de relacionamento interpessoal, está contribuindo com a comunicação terapêutica e desenvolvendo uma assistência de enfermagem Que valorize o paciente como sujeito ativo deste processo.

\section{CONSIDERAÇÕES FINAIS}

A assistência de enfermagem prestada a um paciente deve ser planejada, implementada e avaliada. O planejamento é baseado no levantamento de dados obtidos por meio do histórico de enfermagem, com a entrevista e o exame físico. Com isto, os diagnósticos de enfermagem são identificados e o plano de cuidados elaborado.

A visita de enfermagem é a oportunidade que o profissional de saúde tem para conhecer o paciente, identificando seus problemas e o nível de assistência necessária ao seu cuidado. Este período é a base para o desenvolvimento das outras etapas do processo de enfermagem, daí a importância de se trabalhar estratégias de relacionamento interpessoal Que favoreçam a comunicação terapêutica. Com a identificação do estado de saúde do paciente, o enfermeiro tem compromisso de prestar os cuidados necessários, fornecer uma assistência de enfermagem visando à gestão de Qualidade.

Percebeu-se, neste estudo, Que o relacionamento interpessoal e a comunicação não foram efetivos como deveriam ser, porém, não se pode negá-los nem fugir deles, em razão da sua importância para o processo de enfermagem. Assim, precisa-se trabalhar e desenvolver estratégias de relacionamento interpessoal e com isso efetivar a comunicação entre enfermeiro e paciente, pois o processo de comunicação terapêutica deve ser priorizado como atividade de enfermagem relevante e essencial.

Em relação à identificação do enfermeiro pelo paciente, concluíse Que os pacientes pouco distinguem as enfermeiras dos outros componentes da equipe de enfermagem, o que dificulta a fase de aproximação entre ambos e o início de um relacionamento interpessoal Que contribua para a comunicação terapêutica.

Para alcançar uma comunicação satisfatória e prestar um cuidado humanizado, é preciso Que o enfermeiro deseje envolver-se e acredite Que sua presença é tão importante Quanto a realização de procedimentos técnicos, já Que nem sempre os conhecimentos técnicos objetivos funcionam tão bem, diante de situações de estresse, como os conhecimentos subjetivos que se revelam na comunicação terapêutica. Assim sendo, é preciso reconhecer Que ficar ao lado do paciente para ouvi-lo é uma ação terapêutica e determinante no processo de recuperação da saúde.

Espera-se com este estudo contribuir para Que os profissionais reflitam sobre a importância da utilização das técnicas de relacionamento interpessoal e de comunicação terapêutica na relação de assistência. 


\section{REFERÊNCIAS}

1. Cianciarullo TI. Instrumentos básicos para o cuidar - um desafio para a Qualidade de assistência. I ${ }^{\text {a }}$ ed. São Paulo (SP0: Atheneu; 2003

2. Silva LM, Brasil WV, Barbosa AL. Comunicação não-verbal: reflexões acerca da linguagem corporal. Rev Latino-am enfermagem 2000; 8(4): 52-58.

3. Stefanelli MC. Comunicação com paciente - teoria e ensino. 2a ed. São Paulo (SP): Robe Editorial; 1993.

4. Alfaro LR. Aplicação do processo de enfermagem: um guia passo a passo. $4^{\mathrm{a}}$ ed. Porto Alegre (RS): Artmed; 2000

5. Doenges ME, Moorhouse MF. Diagnóstico e Intervenção em enfermagem. $5^{\text {a }}$ ed. Porto Alegre (RS): Artmed; 2002

6. Peplau HE. Relaciones interpersonales en enfermería: um marco de referência conceptual para la enfermería psicodinámica. Barcelona (ESP): Masson-Salvat; 1990.

7. George JB. Teorias de enfermagem: os fundamentos para a prática profissional. $4^{\mathrm{a}}$ ed. Porto Alegre: Artes Médicas Sul; 2000.

8. Oriá MOB, Moraes LMP, Victor JF. A comunicação como instrumento do enfermeiro para o cuidado emocional do cliente hospitalizado. Rev Eletr Enferm 2004; 6(2): 4l-5.

9. Barbosa APL. Metodologia da pesquisa científica. Fortaleza (CE): UECE; 2001.

10. Minayo MCS. O desafio do conhecimento: peseuisa Qualitativa em saúde. $3^{\text {a }}$ ed. São Paulo (SP): Hucitec/Abrasco; 1994.
11. Ministério da Saúde (BR). Conselho Nacional de Saúde. Resolução n.196, de 10 de outubro de 1996. Dispõe sobre diretrizes e normas reguladoras de pesquisa envolvendo os seres humanos. Bioética 1996; 4: 15-25.

12. Silva AL, Shimizu HE. A relevância da Rede de Apoio ao estomizado. Rev Bras Enferm 2007; 60(3): 307- 11 .

13. Sousa RA, Pessoa SMF, Herculano MMS. A comunicação durante a visita ao leito como fator de Qualidade da assistência de enfermagem In: Anais do $8^{\circ}$ Simpósio Brasileiro de Comunicação em Enfermagem. São Paulo (SP), Brasil; 2002. [citado em 0I Set 2007]. Disponível em: URL: http:// proceedings.scielo.br/scielo.php/script $=$ sci_arttext $\&$ pidMS000000005200200010003 1 \&lng=pt\&nrm-iso

14. Guimarães EMP, Spanol CA, Ferreira E. Utilização do plano de cuidados como estratégia de sistematização da assistência de enfermagem. Rev Cienc Enferm 2002; 8(2):49-58.

15. Trevizan MA, Mendes IAC, Lourenço MR. Aspectos éticos na ação gerencial do enfermeiro. Rev Latino-am Enfermagem 2002; 10(1): 85-9.

16. Hudak CM, Gallo BM. Efeitos da unidade de terapia intensiva sobre o enfermeiro. In: Hudak CM, Gallo BM. Cuidados intensivos de enfermagem: uma abordagem holística. 6 ed. Rio de Janeiro: Guanabara Koogan; 1997.

17. Potter PA, Perry AG. Grande tratado de enfermagem prática: conceitos básicos, teoria e prática hospitalar. 3. ed. São Paulo: Tempo; 2002. 